\title{
A portable wireless power transmission system for video capsule endoscopes
}

\author{
Yu Shi ${ }^{*}$, Guozheng Yan, Bingquan Zhu and Gang Liu \\ 820 Institute, Department of Instrument Science and Engineering, School of Electronic Information \\ and Electrical Engineering, Shanghai Jiao Tong University, Shanghai 200240, China
}

\begin{abstract}
Wireless power transmission (WPT) technology can solve the energy shortage problem of the video capsule endoscope (VCE) powered by button batteries, but the fixed platform limited its clinical application. This paper presents a portable WPT system for VCE. Besides portability, power transfer efficiency and stability are considered as the main indexes of optimization design of the system, which consists of the transmitting coil structure, portable control box, operating frequency, magnetic core and winding of receiving coil. Upon the above principles, the correlation parameters are measured, compared and chosen. Finally, through experiments on the platform, the methods are tested and evaluated. In the gastrointestinal tract of small pig, the VCE is supplied with sufficient energy by the WPT system, and the energy conversion efficiency is $2.8 \%$. The video obtained is clear with a resolution of $320 \times 240$ and a frame rate of 30 frames per second. The experiments verify the feasibility of design scheme, and further improvement direction is discussed.
\end{abstract}

Keywords: Portable wireless power transmission, video capsule endoscope, resonant circuit, frequency stability

\section{Introduction}

Since 2001, when Given Image Company's "Pill Cam" came out, video capsule endoscope (VCE) has attracted attention in the field of medical engineering [1]. Due to the limited capacity of battery, the recording time of VCE is about 8 to 11 hours, which is shorter than the digestion period of human body, so a part of gastrointestinal (GI) tract cannot be inspected. Meanwhile, the image capture rate is 2 to 8 frames per second and the resolution is $256 \times 256$, which would possibly miss the lesion areas [24]. The efficacy of playback functions were compared to reduce the reading time, a high-speed and high-efficiency system was developed, yet none of them reduced the power consumption nor extend the working time of VCE [5, 6]. The insufficient energy supply causes low frame rate, low resolution and short working time, which have impeded the development of VCE in clinical application $[7,8]$.

As an effective solution to this problem, wireless power transmission (WPT) system could replace the batteries: human body is placed in the primary coils, VCE is loaded with the secondary coils and driven by inductive energy, which can record video with high resolution and frame rate [9-12].

Researchers have proposed the model of three-dimensional (3D) transmitting coils and onedimensional (1D) receiving coils [13], but the capsule needs to be positioned to set the direction of

\footnotetext{
* Address for correspondence: Yu Shi, School of Electronic Information and Electrical Engineering, Shanghai Jiao Tong University, Shanghai 200240, China. Tel.: +86 21 34204435; Fax: +86 21 34204435; Email: shiyu359@163.com.
} 
electromagnetic field, which is sensitive due to the mutual inductance among three coils. Besides, propelled capsules and vibratory-actuation capsules driven by the model of 1D transmitting coils and 3D receiving coil have been proposed [14], and some other researches focus on the model and principle of electromagnetic field $[15,16]$. However, these studies are based on the fixed transmitting coil, which means the human body must lie on the bench all the time. Considering the digestion period of human, this model is hard to achieve.

This paper develops a portable wireless power transmitting system, which allows ordinary body actions while supplying energy to VCE. Portability, power transfer efficiency and stability are the main indexes of optimization design. A pair of elliptic solenoids were chosen as the transmitting coils by comparing the structure and weight of three kinds of coil; the uniformity of magnetic field is confirmed by measurement; operating frequency is selected according to the quality factor; transmitting circuit is integrated in a portable box; the magnetic core and winding of receiving coil are determined through tests. Finally, the system is tested on a bench, evaluated through the animal experiments, and the next improvement direction is discussed.

\section{Methods and materials}

\subsection{WPT system overview}

The WPT system, as shown in Figure 1, includes four parts: transmitting circuit, transmitting coil $\left(\mathrm{L}_{1}\right)$, receiving coil $\left(\mathrm{L}_{2}\right)$ and receiving circuit. The control circuit generates an excitation signal at a fixed frequency, which is then amplified to an alternating current $\left(\mathrm{I}_{1}\right)$ by the H-bridge and thus drives the transmitting coil, where the alternating magnetic field is produced. The induced current $\left(\mathrm{I}_{2}\right)$ is stabilized by the rectifier and regulator, and drives the VCE system.

The large distance between coils leads to the weak coupling. Thus the transfer efficiency $(\eta)$ and stability need to be raised for sufficient inductive energy. Meanwhile, considering the human body's action, the portability of transmitting system is also a necessity.

\subsection{Power transfer efficiency}

For the WPT system, energy transfer efficiency $\eta$ can be expressed as [14]:

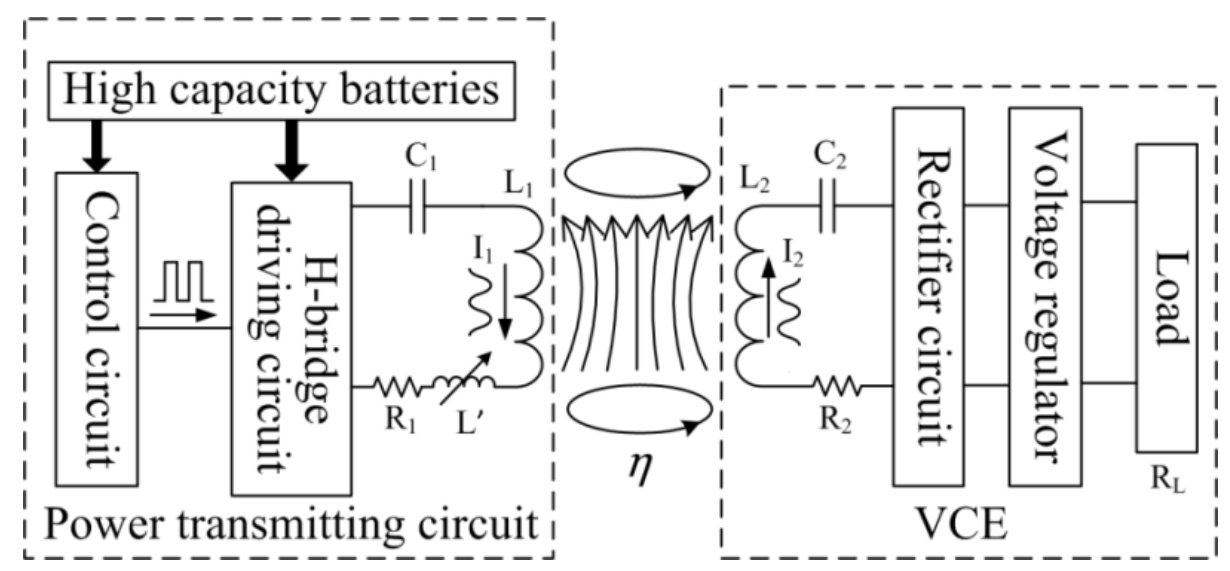

Fig. 1. Structure of WPT system. 


$$
\eta=\frac{\omega B_{1}^{2} S \mu_{2} R_{L} Q_{1}}{\left(R_{2}+R_{L}\right)^{2} L_{1}}, \quad \omega=2 \pi f, \quad R_{2}=\frac{\omega L_{2}}{Q_{2}}
$$

Where $B_{1}$ is the magnetic field intensity, $\mathrm{S}$ is the orthogonal projection area of receiving coil and $\mu_{2}$ is the relative permeability of receiving coil. The angular frequency $\omega$ is corresponding to the operating frequency $f . R_{2}$ and $R_{L}$ are the impedance of receiving coil and load, respectively. $Q_{1}, Q_{2}$ and $L_{1}, L_{2}$ are the quality factors and inductances of transmitting and receiving coil, respectively. Separating these parameters, Eq. (1) can be expressed as:

$$
\eta=B_{1}^{2} R_{L} \cdot X_{1} \cdot X_{2}^{2}, \quad X_{1}=\frac{\omega Q_{1}}{L_{1}}, \quad X_{2}=\frac{S \mu_{2}}{\frac{\omega L_{2}}{Q_{2}}+R_{L}}
$$

$\mathrm{X}_{1}$ and $\mathrm{X}_{2}$ are the influence factors of transmitting coil and receiving coil, respectively. It is obvious that the frequency $(\omega)$, quality factor $\left(\mathrm{Q}_{1}\right)$ and inductance $\left(\mathrm{L}_{1}\right)$ are related to the transmitting coil, and the actual relative permeability $\left(\mu_{2}\right)$, projection area $(S)$, quality factor $\left(\mathrm{Q}_{2}\right)$ and inductance $\left(\mathrm{L}_{2}\right)$ are related to the receiving coil. Thus the system is designed based on $\mathrm{X}_{1}$ and $\mathrm{X}_{2}$.

\section{Design and implementation}

\subsection{Transmitting coils}

\subsubsection{Coils structure}

To control the magnetic field easily, 1D transmitting coils are chosen, which are generally constructed based on solenoid or Helmholtz coils. Helmholtz coils consist of two identical circular plane coils placed symmetrically along a common axis and separated from each other with a distance of the coil radius. Comparing the induced magnetic field, the former is denser, while the latter is more uniform. To combine their advantages, the model of a pair of solenoids is put forward. It consists of two identical solenoid coils with a relative position similar to that of the Helmholtz coils, and the circular coils are transformed into elliptic to alleviate weight and fit the normal human body. These structures of transmitting coils have been shown in Figure 2.

The transmitting coils are constructed with Litz wire (180 strands of AWG 38), and winded for 28 turns of each coil. The shape is firmed by packaging adhesive, which is light and stable. The weight of the prepared coils is $2.1 \mathrm{Kg}$. As shown in Figure 3(a), the long axis is $400 \mathrm{~mm}$, the short axis is 300 $\mathrm{mm}$, the distance between two coils is $200 \mathrm{~mm}$, and the total height is $270 \mathrm{~mm}$. After putting on through the special straps by an adult male, the distance between the coils and the body is less than 15 $\mathrm{mm}$, as shown in Figure 3(b).

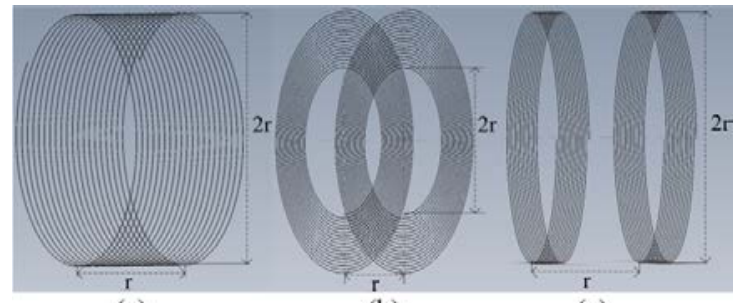

(a)

(b)

(c)

Fig. 2. Three kinds of transmitting coil structure ((a) solenoid coils; (b) Helmholtz coils (c) pair of solenoid coils). 


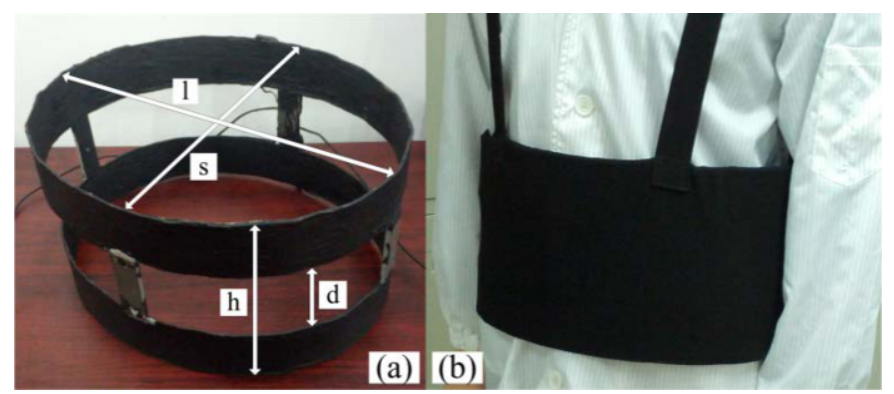

Fig. 3. The portable transmitting coils and the wearing effect ((a) The prepared transmitting coils; (b) Wearing effect of coils; 1: long axis; s: short axis; d: distance between coils; h: total height).

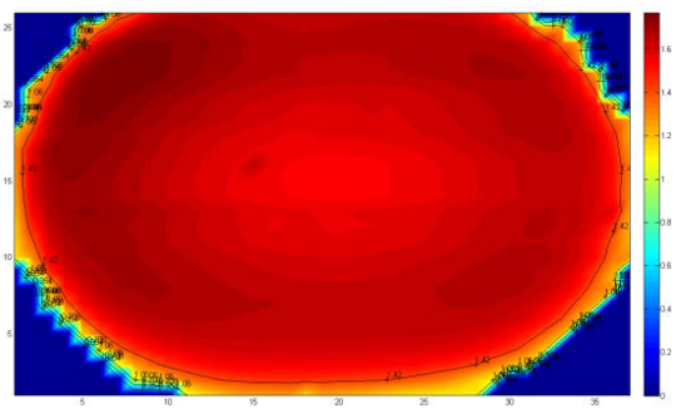

Fig. 4. Distribution of magnetic field.

\subsubsection{Magnetic field distribution}

Magnetic flux heterogeneous distribution could result in energy shortage in somewhere of a given space, thus it is necessary to test the uniformity of magnetic field. The transmitting coils are fixed on the bench and fed with a DC current of $1 \mathrm{~A}$. The magnetic field intensity is then sampled equidistantly $(10 \mathrm{~mm})$ using gauss meter (460 Gaussmeter, LakeShore) on a three-axis instrument table.

Figure 4 shows the magnetic field distribution of the middle radial plane of transmitting coils, indicates the uniformity in the working area of transmitting coils by the contour of 1.42 Gs. It can be noticed that the intensity in the central area is a little less than that in the rim. In this area, the maximal value is $B_{\max }=1.720 \mathrm{Gs}$, the minimal value is $B_{\min }=1.577 \mathrm{Gs}$, and the average value is $B_{0}=1.683$ Gs. According to the definition of uniformity $\gamma=\left(B_{\max }-B_{\min }\right) / \mathrm{B}_{0}, \gamma=8.5 \%$, which is larger than the optimal value $6 \%$ in [7], but considering the geometric shape and the single layer of coils, the uniformity of this transmitting coils is within the allowable range.

\subsubsection{Transmitting frequency}

According to Eq. (2), the influence factor of transmitting coils $\left(X_{1}\right)$ includes frequency $(\omega)$, quality factor $\left(\mathrm{Q}_{1}\right)$ and inductance $\left(\mathrm{L}_{1}\right)$, which can be described as:

$$
\mathrm{X}_{1}=\frac{\omega \mathrm{Q}_{1}}{\mathrm{~L}_{1}}
$$

Through measuring the quality factor and inductance of transmitting coils by impedance analyzer (HITESTER 3532, HIOKI) at the frequency from $100 \mathrm{KHz}$ to $400 \mathrm{KHz}$, the influence factor $\left(\mathrm{X}_{1}\right)$ can be calculated, and the results are shown in Figure 5. 


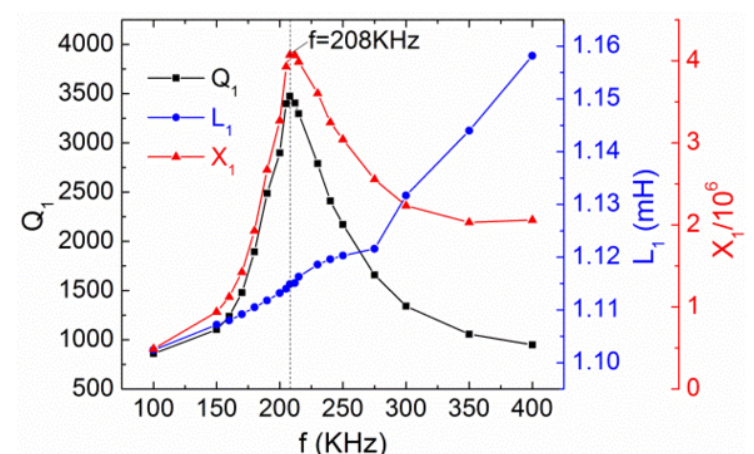

Fig. 5. Changes of three parameters of transmitting coils at different frequencies.

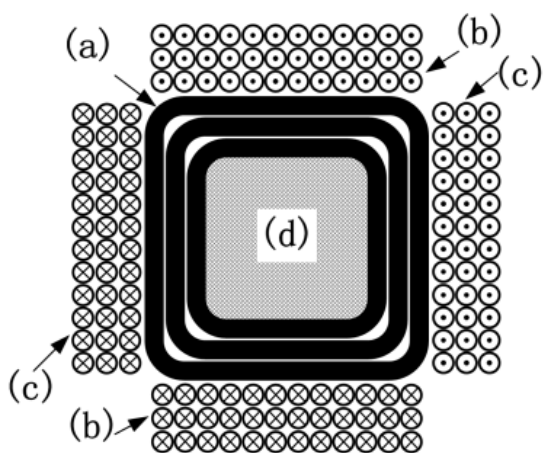

Fig. 6. The cross-sectional view of receiving coils ((a) coil 1; (b) coil 2; (c) coil 3; (d) magnetic core).

More than six orders of magnitude between $\mathrm{L}_{1}$ and the other two parameters, thus $\mathrm{X}_{1}$ is mainly influenced by $\omega$ and $Q_{1}$. The maximum values of $X_{1}$ and $Q_{1}$ appear at the frequency of $208 \mathrm{KHz}$, which leads to the optimum value of energy transfer efficiency $(\eta)$ in the part of transmitting coils, based on Eq. (2). Therefore, $208 \mathrm{KHz}$ is chosen as the transmitting frequency.

\subsection{Receiving coils}

A 3D receiving coil is designed to ensure inductive energy while the orientation of coil changes. It is actually a combinatory of three coils, which are geometrically orthogonal to each other and winded on a common ferrite core layer by layer. The cross-sectional view is shown in Figure 6.

\subsubsection{Magnetic core}

The definition of induced electromotive force can be described as:

$$
\varepsilon=\mathrm{I}_{\mathrm{m}} \mathrm{B}_{1} \omega \mathrm{S} \mu
$$

Where $I_{m}$ is the amplitude of transmitting current, $B_{1}$ is the magnetic field intensity, $S$ is the orthogonal projection area, $\omega$ is the frequency and $\mu$ is the permeability. Thus the area coefficient $(\xi)$ is presented, which synthesizes $\mathrm{S}$ and $\mu$, for evaluating the actual relative permeability. The $\xi$ ensures the inductive energy of each coil is approximately equal, which is described as:

$$
\left\{\begin{array}{l}
\xi_{\mathrm{i}}=\mathrm{S} \cdot \mu=\mathrm{S}_{\text {air }} \cdot \mu_{0}+\mathrm{S}_{\text {core }} \cdot \mu_{\mathrm{c}} \\
\xi_{1}=\xi_{2}=\xi_{3} \\
\mathrm{i}=1,2,3
\end{array}\right.
$$

Where $S_{\text {air }}$ and $S_{\text {core }}$ are the surrounded area of air and core respectively, $\mu_{0}$ and $\mu_{\mathrm{c}}$ are the relative permeability of air and core, respectively. The area coefficients $\left(\xi_{\mathrm{i}}\right)$ correspond to the influence factor $\mathrm{X}_{2}$, which reflect the degree of the actual permeability of $1 \mathrm{D}$ coil, and are differentiated by the subscript i. Thus the Eq. (4) can be obtained as follows:

$$
\varepsilon=\mathrm{I}_{\mathrm{m}} \mathrm{B}_{1} \omega \cdot \xi
$$


It is obvious that when the transmitting parameters are determined, the induced electromotive force $(\varepsilon)$ is proportional to the area coefficient $(\xi)$. Winding the cores to the calculated sizes, placing them in the same transmitting condition and testing $\varepsilon$, and $\xi$ can be compared. Considering the error caused by manual operations, each size of $1 \mathrm{D}$ coil is prepared three times, and the transmitting currents are 0.5 A, $0.8 \mathrm{~A}$ and $1.2 \mathrm{~A}$, respectively. The test result is shown in Figure 7.

The curves in Figure 7 show that the proportion and changing trend of $\varepsilon$ keep consistent with $\xi$, which proves the linear relationship between them. There are maximum values of $\varepsilon$ and $\xi$ when the diameter of core is $6.0 \mathrm{~mm}$, the size of which is chosen as the magnetic core of receiving coils.

\subsubsection{Winding strands and turns}

According to the Eq. (2), the influence factor of receiving coils is described as:

$$
\mathrm{X}_{2}=\frac{\mathrm{S} \mu_{2}}{\frac{\omega \mathrm{L}_{2}}{\mathrm{Q}_{2}}+\mathrm{R}_{\mathrm{L}}}
$$

Where the quality factor $\left(\mathrm{Q}_{2}\right)$ and inductance $\left(\mathrm{L}_{2}\right)$ are the related parameters of receiving coils, when the operating frequency and magnetic core are chosen. These two parameters are both influenced by the winding line. Because of the explicit size of coils and core, the collocations of winding strands $(\mathrm{N})$ and turns (n) are fixed. The relationships of these parameters can be described as:

$$
\left\{\begin{array}{c}
\mathrm{L}_{2} \propto \mathrm{n}, \mathrm{L}_{2} \propto 1 / \mathrm{N} \\
\mathrm{R}_{2} \propto \mathrm{n}, \mathrm{R}_{2} \propto 1 / \mathrm{N} \\
\mathrm{V}(\mathrm{N}, \mathrm{n}) \sim \mathrm{Q}_{2} \sim \mathrm{X}_{2}
\end{array}\right.
$$

There must be a collocation of $\mathrm{N}$ and $\mathrm{n}$, which leads to the maximum values of $\mathrm{Q}_{2}$. Nine kinds of Litz wire are chosen for winding to $3 \mathrm{D}$ coils, and the quality factors $\left(\mathrm{Q}_{2}\right)$ of coils are measured (HITESTER 3532, HIOKI). The coils are connected to resistance of $139 \Omega$ (the equivalent resistance of VCE) and tested in the transmitting coils $(208 \mathrm{KHz}, 1 \mathrm{~A})$, so that the $\mathrm{P}_{2}$ can be obtained. As shown in Figure 8, the trend of receiving power is corresponding to the quality factor, which proves that the quality factor directly effects on the receiving energy and efficiency. There are maximum values of $\mathrm{Q}_{2}$ and $\mathrm{P}_{2}$ when the Litz wire is 12-strand, which is chosen as the winding wire of receiving coils.

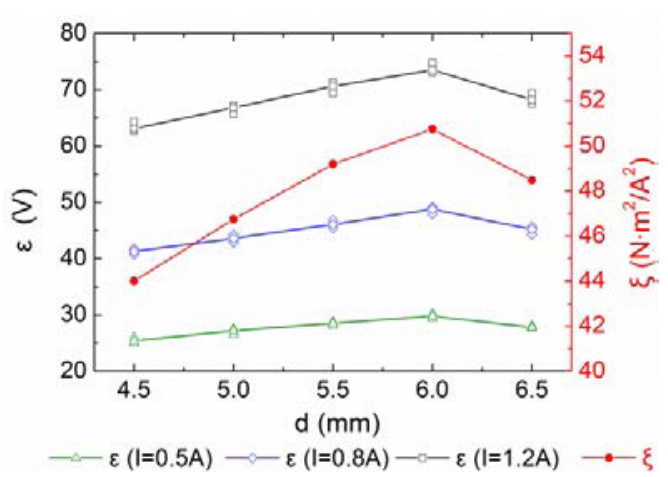

Fig. 7. Induced electromotive forces and area coefficients in different transmitting condition (d: diameter of core; $\varepsilon$ : induced electromotive force; $\xi$ : area coefficient).

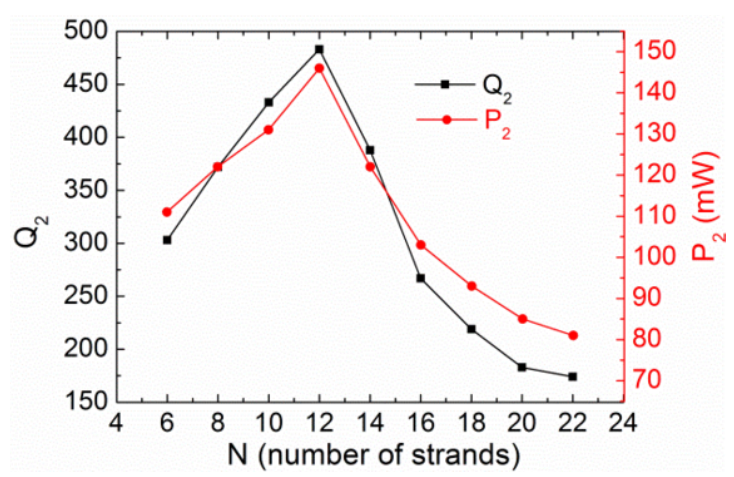

Fig. 8. Quality factor and receiving power of $3 \mathrm{D}$ coils winded by Litz wires. 


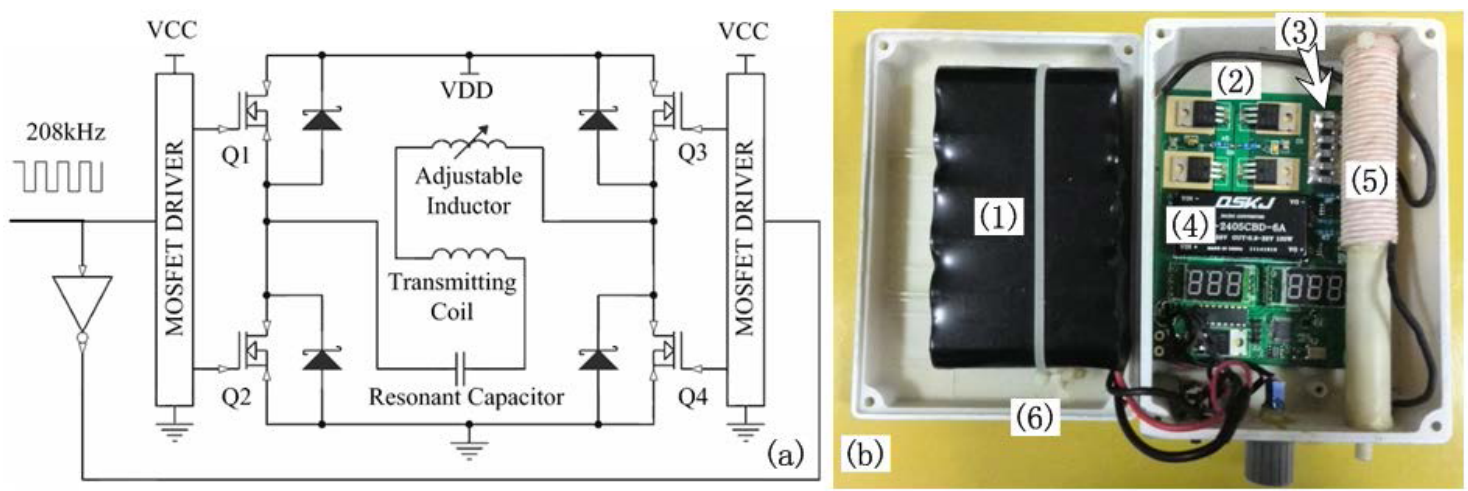

Fig. 9. Schematic of the transmitting driver and the portable realization ((a) The schematic of the transmitting driver; (b) The overview of control box; (1) Lithium-ion batteries; (2) Full-bridge circuit; (3) Resonant chip capacitors; (4) DC/DC converter; (5) Adjustable inductance; (6) The case).

\subsection{Transmitting circuit}

To induce a time-vary magnetic field, a full-bridge form of class D inverter is adopted because of the flexibility and the deformation. This class D inverter is composed from four MOSFETs (Q1, Q2, Q3, Q4 in Figure 9(a)), and driven by two MOSFETs with mutual reverse signals. The square wave signal is produced by the timing generator, and then amplified into a rectangular wave current in the LC tank, which consists of the transmitting coil, resonant capacitor and adjustable inductor in series. Both the Q value and withstand voltage capacity of the resonant capacitors should be as high as possible to reduce the power dissipation.

As shown in Figure 9(b), the control box is powered by lithium-ion batteries; the MOSFETs are fixed on the board for dissipating heat; the resonant chip capacitors are chosen for saving space; the DC/DC converter is more stable than complicated circuit; in addition, the adjustable inductance is placed in the box to keep a constant operating frequency. The size of the box is $140 \mathrm{~mm} \times 100 \mathrm{~mm} \times 60$ $\mathrm{mm}$, and the weight of that is about $1 \mathrm{Kg}$, which is easy to be carried.

\section{Tests and experiments}

\subsection{Frequency stability}

Slight movement of body could cause little change of inductance, which leads to transmitting frequency drift in a small range. Because of the positive correlation between frequency offset and transmitting power drop (as shown in Figure 5), the drift degree of frequency can be evaluated by the reduction degree of current in a fixed driven voltage.

As shown in Figure 10, when the resonant frequency $\left(\mathrm{f}_{0}\right)$ is set to $208 \mathrm{KHz}$ by the signal generator (RIGOL 3101A), the transmitting coils are driven by the current of $1 \mathrm{~A}$. Keeping the driving voltage and changing the frequency every other $0.1 \mathrm{KHz}$, it is found that the current remains about the same in the range of $\mathrm{f}_{0} \pm 0.5 \mathrm{KHz}$, which is caused by the reflection effect of electromagnetic wave. Therefore, influence of slight movement can be ignored unless the driving current drops sharply. 


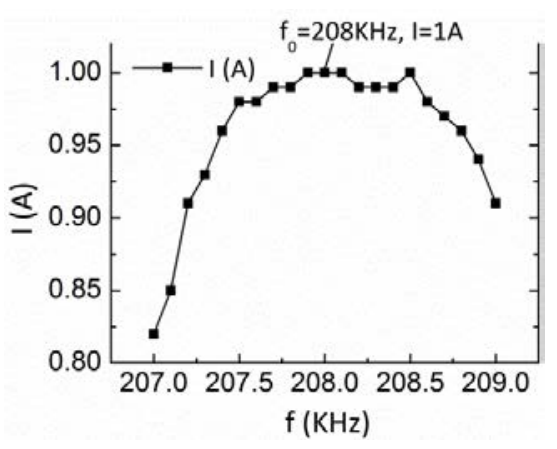

Fig. 10. The changes of driving current near resonance point.

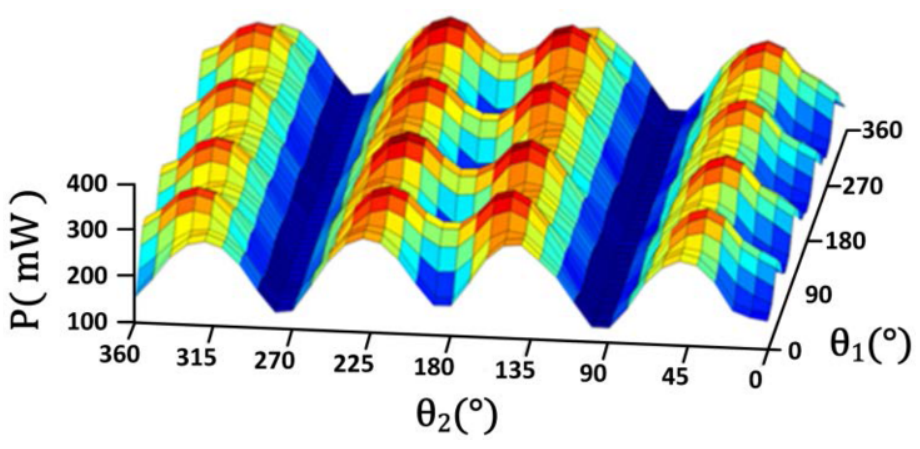

Fig. 11. Receiving power on the load.

\subsection{Receiving power}

To test whether the receiving power is enough, the transmitting coils are driven by the current of $207 \mathrm{KHz}$ (the resonant frequency is $208 \mathrm{KHz}$, to simulate the worst frequency drift condition), and a two-axis turntable is put in the center of it, where the magnetic flux density is minimum. Fixing the 3D receiving coils on the bench and pivoting in two dimensions by the spin motors, the receiving power on the load (pure resistance of $139 \Omega$, the equivalent resistance of VCE) can be measured.

Figure 11 shows the variations in the receiving power when pivoting angles $\left(\theta_{1}, \theta_{2}\right)$ change from $0^{\circ}$ to $360^{\circ}$ by step of $10^{\circ}$ each. When a dimension of receiving coils is perpendicular to the direction of the magnetic field, the troughs of the receiving power appear, and the value is approximately $80 \mathrm{~mW}$. On the contrary, the peaks appear when each dimension has a corresponding angle of $55^{\circ}$ with the direction of magnetic field, and the value is approximately $350 \mathrm{~mW}$.

\subsection{Experiment on platform}

In the air medium, the minimum transmitting current is $0.60 \mathrm{~A}$ with a driving voltage of $3.67 \mathrm{~V}$, while the power transfer efficiency is $3.5 \%$. The VCE system works normally; the effective communication distance is less than $2.0 \mathrm{~m}$, as shown in Figure 12(a).

As for the medium of pork $(10 \mathrm{Kg})$, the minimum transmitting current is $0.63 \mathrm{~A}$ with driving voltage of $3.89 \mathrm{~V}$, and the power transfer efficiency is $3.2 \%$. The VCE works normally, and the effective communication distance is less than $1.8 \mathrm{~m}$, as shown in Figure 12(b).

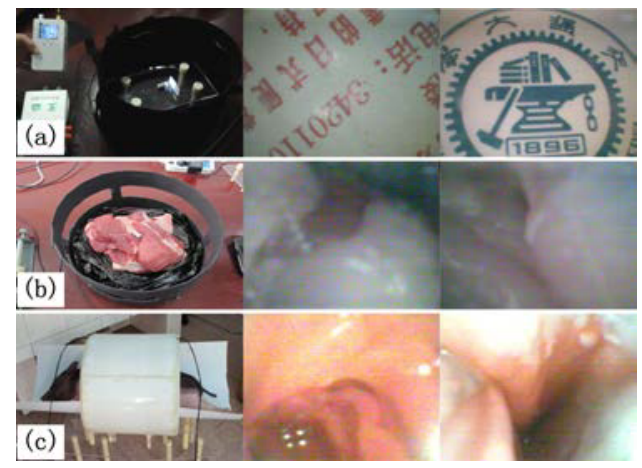

Fig. 12. VCE on the portable WPT platform ((a) In the air medium; (b) In the medium of pork; (c) In the living pig). 
When the transmitting coils are put on an anesthetized pig $(20 \mathrm{Kg})$ and the VCE is swallowed 8 minutes later, the VCE arrives in the stomach and sends video. The minimum transmitting current is 0.67 A with driving voltage of $4.16 \mathrm{~V}$, while the power transfer efficiency is $2.8 \%$. The VCE works normally, and clear images of GI tract can be received with the effective communication distance less than $1.3 \mathrm{~m}$, as shown in Figure 12 (c). In addition, when the pig is moved in the transmitting coils, frequency drifts in some attitudes, meanwhile the driving current decreases about $0.2 \mathrm{~A}$ and the video signal is unstable, which can be restored by turning the adjustable inductance.

In all of these experiments, the resolution of the images is $320 \times 240$, and the format of the video is NTSC with the frame rate of 30 frames per second.

\section{Discussions and conclusions}

This paper shows important steps toward a portable WPT system, as an alternate of the fixed one. Portability, efficiency and stability are the main indexes of design, which include the transmitting coils, operating frequency, 3D receiving coils and relevant circuit.

Considering portability and controllability, an elliptic pair of solenoids is chosen as the transmitting coil, and the alternating magnetic field can cover the GI tract uniformly. Meanwhile, the strength and distribution of the magnetic field prove that the structure of coil is feasible via measurement. In addition, the transmitting circuits are analyzed, and improved for portability.

Because of the weak coupling limitation, energy transfer efficiency is low. According to the formulas, the influence factors of transmitting coils and receiving coils are presented. The former decides the proper operating frequency by quality factor; the latter can be summarized as the area coefficient and winding strands of receiving coils, for sufficient and stable energy.

When the actual frequency drifts around the resonant frequency within $0.5 \mathrm{KHz}$, the transmitting current remains almost the same due to the reflection effect of electromagnetic wave. A relatively narrow fluctuation of the inductance changes the frequency a little, and has even smaller influence on the transmitting power. Thus the slight movement of body can be ignored; meanwhile, the sharply drop of driving current caused by environment can be offset by turning the adjustable inductance.

The induced energy varies with the position of 3D receiving coils, which reaches minimum value when a $1 \mathrm{D}$ coil is perpendicular to the direction of the magnetic field, and reaches maximum value when each coil has a corresponding angle of $55^{\circ}$ with the direction of magnetic field. The variation range of the receiving energy is sufficient to the VCE and acceptable to the receiving circuit.

The prepared system is tested in three environments. Comparing the results above, the power transmitting efficiency gradually decreases from $3.5 \%$ to $2.8 \%$ with the medium changes from air to living animal, which means the effect of medium on the LC resonance circuit enhances.

The feasibility of portable WPT system has been proved and evaluated. However, the efficiency of it is lower than the fixed one, because the limited space in the control box intensifies the electromagnetic interference; the size and weight of the transmitting coils are inapposite for larger waist; in addition, the video signal is not ideal when the communication is out of range. Thus the future work will be focused on the electromagnetic shielding of transmitting circuit, new structures and materials for transmitting coils, and antennas on body surface to raise the receiving sensitivity. The system will be experimented on animal to check the feasibility more times.

\section{Acknowledgments}


This work has been supported by the National Natural Science Foundation of China (NSFC) (Nos. 60875061, 31170968, 30800235).

\section{References}

[1] G. Iddan, G. Meron, A. Glukhovsky, et al., Wireless capsule endoscopy, Nature 405 (2000), 417.

[2] T. Khan, R. Shrestha, M.S. Imtiaz, et al., Colour-reproduction algorithm for transmitting variable video frames and its application to capsule endoscopy, Healthcare Technology Letters 2 (2015), 52-57.

[3] L.J. Sliker and G. Ciuti, Flexible and capsule endoscopy for screening, diagnosis and treatment, Expert Review of Medical Devices 11 (2014), 649-666.

[4] P. Valdastri, M. Simi and R.J. Webster III, Advanced technologies for gastrointestinal endoscopy, Annual review of biomedical engineering 14 (2012), 397-429.

[5] M.R. Basar, M.Y. Ahmad, J. Cho, et al., Application of Wireless Power Transmission Systems in Wireless Capsule Endoscopy: An Overview, Sensors 14 (2014), 10929-10951.

[6] K. Kim, S. Yun, S. Lee, et al., A design of a high-speed and high-efficiency capsule endoscopy system, IEEE Transactions on Biomedical Engineering 59 (2012), 1005-1011.

[7] Z. Jia, G. Yan, H. Liu, et al., The optimization of wireless power transmission: design and realization, The International Journal of Medical Robotics and Computer Assisted Surgery 8 (2012), 337-347.

[8] G. Ou, N. Shahidi, C. Galorport, et al., Effect of longer battery life on small bowel capsule endoscopy, World Journal of Gastroenterology 21 (2015), 2677-2682.

[9] M.L. Kung and K.H. Lin, Enhanced analysis and design method of dual-band coil module for near-field wireless power transfer systems, IEEE Transactions on Microwave Theory and Techniques 63 (2015), 821-832.

[10] R. Carta and G. Tortora, Wireless powering for a self-propelled and steerable endoscopic capsule for stomach inspection, Biosensors and Bioelectronics 25 (2009), 845-851.

[11] H. Li, G. Yan and P. Gao, A method for improving the wireless power transmission efficiency of an endoscopic capsule based on electromagnetic localization and synthesis of magnetic field vector, Proc. IMechE Part C: J. Mechanical Engineering Science 224 (2010), 1463-1471.

[12] I.M. Gralnek, J.Y. Ching, I. Maza, et al., Capsule endoscopy in acute upper gastrointestinal hemorrhage: A prospective cohort study, Endoscopy 45 (2013), 12-19.

[13] G. Pan, W Xin, G. Yan, et al., A video wireless capsule endoscopy system powered wirelessly: Design, analysis and experiment, Measurement Science \& Technology 22 (2011), 2572-2575.

[14] B. Zhu, G. Yan, G. Liu, et al., Design of a dual-head video capsule endoscopy system based on wireless power transmission technology, Journal of Medical and Biological Engineering 2 (2014), 1-25.

[15] A.V. Gossum, Wireless capsule endoscopy of the large intestine: a review with future projections, Current Opinion in Gastroenterology 30 (2014), 472-476.

[16] H. Neumann, L.C. Fry and M.F. Neurath, Review article on current applications and future concepts of capsule endoscopy, Digestion 87 (2013), 91-99. 